\title{
A Novel Stochastic Combination of 3D Texture Features for Automated Segmentation of Prostatic Adenocarcinoma from High Resolution MRI
}

\author{
Anant Madabhushi ${ }^{1}$, Michael Feldman ${ }^{1}$, Dimitris Metaxas $^{2}$, Deborah Chute $^{1}$, \\ and John Tomaszewski ${ }^{1}$ \\ 1 University of Pennsylvania, Philadelphia, PA 19104 \\ $\{$ anantm@seas. upenn.edu \\ 2 Rutgers the State University of New Jersey, Piscataway, NJ 08854 \\ $\{$ dnm@cs.rutgers.edu $\}$
}

\begin{abstract}
In this work, we present a new methodology for fully automated segmentation of prostatic adenocarcinoma from high resolution MR by using a novel feature ensemble of 3D texture features. This work represents the first attempt to solve this difficult problem using high resolution MR. The difficulty of the problem stems from lack of shape and structure in the adenocarcinoma. Hence, in our methodology we compute statistical, gradient and Gabor filter features at multiple scales and orientations in 3D to capture the entire range of shape, size and orientation of the tumor. For an input scene, a classifier module generates Likelihood Scenes for each of the 3D texture features independently. These are then combined using a weighted feature combination scheme. The ground truth for quantitative evaluation was generated by an expert pathologist who manually segmented the tumor on the MR using registered histologic data. Our system was quantitatively compared against the performance of the individual texture features and against an expert's manual segmentation based solely on visual inspection of the $4 \mathrm{~T}$ MR data. The automated system was found to be superior in terms of Sensitivity and Positive Predictive Value.
\end{abstract}

\section{Introduction}

Prostatic adenocarcinoma is the most common malignancy of men with an estimated 189,000 new cases in the USA in 2002 and is the most frequently diagnosed cancer among men. Prostate cancer is most curable when detected early. Current screening for prostate cancer relies on digital rectal exam and serum prostate specific antigen (PSA) levels [2] Definitive diagnosis of prostate carcinoma, however, rests upon histologic tissue analysis, most often obtained via needle biopsy guided by transrectal ultrasound (TRUS). Magnetic resonance imaging of the prostate gland is a relatively new technique for staging prostate cancer and it has been shown to produce better tissue contrast between cancers in the peripheral zone compared to ultrasound [2]. The $1.5 \mathrm{~T}$ MR has been shown to be 
more sensitive at detecting seminal vesicle invasion than transrectal ultrasound. Researchers at the University of Pennsylvania have been exploring the use of high resolution MR imaging of prostatectomy specimens using a $4 \mathrm{~T}$ whole body magnet. MR imaging under a 4T magnet has been shown to allow for greater separation of normal, benign prostatic hyperplasia, and carcinoma compared to $1.5 \mathrm{~T}$.

While researchers have proposed Computer Aided Detection (CAD) systems for automatically detecting breast and lung cancers, no automated system exists for detecting prostatic adenocarcinoma in MR. Given the high incidence of prostate cancer in men, such a system would be extremely useful. Our motivation behind this work was,

(i) The prospect of creating accurate CAD techniques that could show the advantages of using high resolution MR over ultrasound for detecting Prostatic Adenocarcinoma.

(ii) Increasing the probability of detecting cancer using blind sextant biopsies and reducing the number of needle insertions required to find cancerous tissue.

(iii) Remove the subjectivity of inter- and intra-observer interpretation and objectively determine the presence and location of cancer in the MR scan.

Visual identification of small prostatic tumors is confounded by the fact that several benign features have overlapping texture and intensity appearance including clusters of atrophic glands and areas of prostatic stromal over-growth. The difficulty of the problem is exacerbated by lack of structure and shape in the adenocarcinoma. Texture operators have been widely used for medical image segmentation. While 2D texture has been extensively studied, there has been very little work done in the use of $3 \mathrm{D}$ volumetric texture operators in medical image segmentation. First order statistics depend only on the individual pixel values. Second order statistics are calculated from the probability of observing a pair of pixel values in the image that are separated by some displacement vector. To build a system that can discriminate textures at least as well as humans do, we need to take into account both first and second-order statistics. Gradient operators have been shown to characterize micro-textures well [1]. It has been suggested that gradient operators show more consistent behavior as a descriptor of pathologies than co-occurrence matrices [1]. While the 2D Gabor filter has been widely used for pattern recognition problems, the $3 \mathrm{D}$ version has found limited usage in segmentation; being used mostly for motion estimation. In this paper we extend the 2D Gabor transform to 3D and compute it at multiple scales and orientations. The use of different classes of 3D texture features would enable us to capture the entire range of variation in size, shape and orientation of the cancer.

Both empirical observations and specific machine learning applications confirm that a given feature outperforms all others for a specific subset of the input data, but it is unusual to find a single feature achieving the best results on the overall problem domain. In this work we present a weighted feature combination scheme that works by minimizing a novel cost function. By fusing the orthogonal information from the different 3D features our automated system outperforms 
not only the individual features, but also a human expert in terms of Sensitivity and Positive Predictive Value (PPV).

The organization of the paper is as follows. In Section 2 we discuss past work. Sections 34 and 5 describe our methodology. In Section 6 we present our results (Qualitative and Quantitative). Finally in section 7 we present our conclusions.

\section{Previous Work}

While 2D texture operators have been widely used in image processing, surprisingly little attention has been paid to 3D texture operators. Previous work in $3 \mathrm{D}$ texture segmentation has comprised of applying 2D methods to a volume on a per slice basis. This approach however does not exploit much of the embodied information along the axial direction in the stack of aligned images.

Past related work in automatically detecting prostatic cancer has comprised of using first order moment features [6] or co-occurrence matrices 37] to determine benignity or malignancy of a manually segmented region in $2 \mathrm{D}$ ultrasound images. Our work is novel in the following ways,

(i) Unlike previous semi-automated approaches for $2 \mathrm{D}$ ultrasound, this is the first attempt at detecting prostatic tumor on high resolution MR.

(ii) The use of 3D texture operators directly within the MR volume enables us to detect a greater range of variation in appearance, size and orientation of the cancer.

(iii) Combining the 3D texture features by using an optimally weighted feature ensemble employing a novel cost function which is able to outperform a human expert.

\section{MR Data Generation and Experimental Design}

Immediately following radial prostatectomy, the prostate glands are embedded in $2 \%$ agar $(30 \mathrm{mM} \mathrm{NaCl})$ at $50^{\circ} \mathrm{C}$ and cooled to $4^{\circ} \mathrm{C}$ to solidify in a small Plexiglas box. The gland is then imaged using a $4 \mathrm{~T}$ magnet using $2 \mathrm{D}$ fast spin echo. While T1-weighted images are generally prescribed for delineation for the capsule and peri-glandular fatty regions, they however lack structural contrast within the prostate. Hence T2-weighting is preferable. MR and histologic slices are maintained in the same plane of section by both leveling the gland in the $x, y$ and $z$ planes while in the MR magnet as well as by using a rotary knife to cut serial sections of the embedded gland starting at its square face. The prostate gland is serially sliced at $1.5 \mathrm{~mm}$ thick intervals (correlating to $2 \mathrm{MRI}$ slices) and $4 \mu \mathrm{m}$ thick histologic sections are produced by quartering each 1.5 $\mathrm{mm}$ slice. An expert pathologist manually segmented out tumor regions on the $4 \mathrm{~T}$ MR slices, by visually registering the MR with the histology on a per-slice basis. Distinctive features seen on each image slice (histology, MR and gross photographs) were used to manually register and correlate the MR slices with the histologic whole-mount composites. 


\section{Feature Extraction}

On account of the lack of structure and shape in prostatic adenocarcinoma, texture operators are required for segmentation. Texture features that could discriminate between benign and malignant prostatic neoplasia in MR images however, have not as yet been identified. Our choice of texture features, i.e. statistical, gradient and steerable filters were determined by the desire to capture the entire range of variability in appearance, size and orientation of the prostatic neoplasia. Before feature extraction the MR scene is first corrected for intensity inhomogeneity and subsequently standardized to account for the acquisition-toacquisition signal variations inherent in MR images [5].

\subsection{Statistical Texture Features}

We compute both first and second-order statistics in 3D. The first order statistical features: intensity, median, standard and average deviation are computed within a $\mathcal{K} \times \mathcal{K} \times \mathcal{K}$ cube centered at every voxel within the image volume at two different scales $(\mathcal{K}=3,5)$. Co-occurrence matrices were originally proposed by Haralick 4] for 2D images. For $G$ number of gray-levels in the image, the size of the co-occurrence matrix is $G \times G$. The entry $(i, j)$ in the matrix is the number of occurrences of the pair of gray levels $i$ and $j$. The 2D formulation is easily extended to $3 \mathrm{D}$ and given as,

$$
\mathcal{P}_{d \psi \phi}=\left|\left\{\left((r, s, t),\left(r^{\prime}, s^{\prime}, t^{\prime}\right)\right): I_{c s}(r, s, t)=i, I_{c s}\left(r^{\prime}, s^{\prime}, t^{\prime}\right)=j\right\}\right|
$$

where $(r, s, t),\left(r^{\prime}, s^{\prime}, t^{\prime}\right) \in \mathcal{M} \times \mathcal{N} \times \mathcal{L},\left(r^{\prime}, s^{\prime}, t^{\prime}\right)=(r+d \cos \psi \cos \phi, s+d \sin \psi \cos \phi$, $u+d \sin \phi+\eta)$ and $|\cdot|$ is the cardinality of the set. $\mathcal{M}, \mathcal{N}, \mathcal{L}$ are the dimensions of $I_{c s}$, the corrected and standardized image volume, $d$ is the displacement, $\phi, \psi$ are the orientations in 3D and $\eta$ accounts for the anisotropy along the $z$-axis. In our system, we set $d$ to 1 and $\psi=\phi$ to $\frac{\pi}{2}$. Five texture features as proposed by Haralick [4] were computed from the co-occurrence matrix at every voxel in the image volume; energy, entropy, contrast, homogeneity and correlation.

\subsection{Gradient Features}

We compute both the directional gradient and gradient magnitude in 3D. The directional gradient image $I_{c s}^{d g}$ is computed as,

$$
I_{c s}^{d g}=\frac{-\hat{Q}}{\|\hat{Q}\|} \quad \text { where } \quad \hat{Q}=\left[\frac{\partial I_{c s}}{\partial x}, \frac{\partial I_{c s}}{\partial y}, \frac{\partial I_{c s}}{\partial z}\right]\left[\tilde{n}_{x}, \tilde{n}_{y}, \tilde{n}_{z}\right]^{\mathbf{T}}
$$

$\hat{Q}$ is a $3 \mathrm{D}$ vector scene representing the summation of the directional gradients, $\frac{\partial I_{c s}}{\partial x}, \frac{\partial I_{c s}}{\partial y}, \frac{\partial I_{c s}}{\partial z}$ correspond to the image gradients along the $x, y$ and $z$ axes respectively and $\tilde{n}_{x}, \tilde{n}_{y}, \tilde{n}_{z}$ are the normalized derivatives. The gradient magnitude $I_{c s}^{g m}$ is $\|\hat{Q}\|$. In computing the gradient along the z-axis we factored in the anisotropic inter-slice separation. 


\subsection{Steerable Filters}

The 3D MR volume can be regarded as the weighted sum of 3-D Gabor functions of the form,

$$
I_{c s}(x, y, z)=\frac{1}{2^{\frac{3}{2}} \sigma_{x} \sigma_{y} \sigma_{z}} e^{\frac{-1}{2}\left[\frac{x^{2}}{\sigma x^{2}}+\frac{y^{2}}{\sigma y^{2}}+\frac{z^{2}}{\sigma z^{2}}\right]} \cos \left(2 \pi u_{0} x\right)
$$

where $u_{0}$ is the frequency of a sinusoidal plane wave along the $x$-axis, and $\sigma_{x}, \sigma_{y}$ and $\sigma_{z}$ are the space constraints of the Gaussian envelope along the $x, y$ and $z$ axes respectively. The set of self-similar Gabor filters are obtained by appropriate rotations and scalings of $I_{c s}(x, y, z)$ through the generating function [8]:

$$
g_{m n}(x, y, z)=a^{-m} g\left(x^{\prime}, y^{\prime}, z^{\prime}\right), a \geq 1
$$

where $g_{m n}(x, y, z)$ is the rotated and scaled version of the original filter, $a$ is the scale factor, $n=0,1, \ldots, N-1$ is the current orientation index, $N$ is the total number of orientations, $m=0,1,2 \ldots, M-1$ is the current scale index, $M$ is the total number of scales, and $x^{\prime}, y^{\prime}$ and $z^{\prime}$ are the rotated coordinates:

$$
x^{\prime}=a^{m}(x \cos \theta+y \sin \theta), y^{\prime}=a^{m}(-x \sin \theta+y \cos \theta), z^{\prime}=a^{m} z
$$

where $\theta=\frac{n \pi}{N}$ is the orientation, $a=\left(\frac{U_{h}}{U_{l}}\right)^{\frac{1}{M-1}}$ where $U_{h}, U_{l}$ correspond to the upper and lower center frequencies of interest. We used a total of 18 different filter channels, corresponding to 6 orientations and 3 scales. For each Gabor filter channel the standard deviation $\sigma_{m n}$ within a small neighborhood centered at each voxel $\mathbf{x}$ was computed, resulting in a feature vector: $f(\mathbf{x})=\left[\sigma_{m n}(\mathbf{x}) \mid m=\{0, . ., \mathrm{M}-1\}\right.$, $n=\{0, . ., N-1\}]$. Spearman's $(\rho)$ rank correlation analysis of the different filtered outputs within each scale and orientation and across different scales and orientations was performed. Only those pairs of filters that had low correlation were assumed to be orthogonal and retained.

In all, 32 features from the three different texture classes were computed.

\section{Feature Classification}

This module comprises of three blocks, (i) a training block in which the probability density functions (pdf's) for each of the 3D features is built, (ii) an individual feature classifier block for generating Likelihood Scenes for each of the 3D features and (iii) a feature combination block in which these different Likelihood Scenes are combined.

\subsection{Training}

Training is performed off-line and only once. In all, 15 slices from 2 different prostate glands were used for training. The manually segmented tumor regions on the MR were used as masks for generating pdf's for each of the $3 \mathrm{D}$ features. To 
each one of the training images the different 3D texture operators were applied and different Feature Scenes were generated. All voxels within the tumor mask regions in each of the Feature Scenes were scaled into a common range in order to build the pdf's. For each voxel within the tumor mask the response of each $3 \mathrm{D}$ texture operator was noted and the corresponding value incremented in the corresponding feature histogram.

\subsection{Individual Feature Classifier}

For a voxel $\mathbf{x}$ in the input scene we used Bayesian inference [9] to assign a likelihood of malignancy based on each of the $K$ texture features $\left(f_{\gamma=1, \ldots, K}\right)$ independently. For every input image, a Likelihood Scene corresponding to each $3 \mathrm{D}$ feature is generated.

$$
P\left(\mathbf{x} \in \omega_{\varrho} \mid f_{\gamma}\right)=P\left(\mathbf{x} \in \omega_{\varrho}\right) \frac{p\left(f_{\gamma} \mid \mathbf{x} \in \omega_{\varrho}\right)}{p\left(f_{\gamma}\right)}
$$

where the a-posteriori probability of observing the class $\omega_{\varrho}$ given the feature $f_{\gamma}$ is given by $P\left(\mathbf{x} \in \omega_{\varrho} \mid f_{\gamma}\right) . P\left(\mathbf{x} \in \omega_{\varrho}\right)$ is the a-priori probability of observing the class $\omega_{\varrho}, p\left(f_{\gamma} \mid \mathbf{x} \in \omega_{\varrho}\right)$ is the conditional density obtained from the training models. $p\left(f_{\gamma}\right)=\sum_{\varrho=1}^{c} p\left(f_{\gamma} \mid \mathbf{x} \in \omega_{\varrho}\right) P\left(\mathbf{x} \in \omega_{\varrho}\right)$ is treated as a constant with $c$ refering to the number of classes. For our problem, $c=2$, i.e. tumor and non-tumor. Assuming an equal likelihood that voxel $\mathbf{x}$ could be cancer or not, $P\left(\mathbf{x} \in \omega_{\varrho}\right)=0.5$ and can be regarded as a constant. The independence assumption was used to combine the different Gabor filter channels and the second order co-occurrence features to obtain a single Gabor and a co-occurrence feature respectively.

\subsection{Feature Combination}

Multiple classifier systems are often practical and effective solutions for difficult pattern recognition problems since they seek to exploit the variance in behavior of the base learners. They can be divided into two types:

(i) Non-generative methods: These confine themselves to a set of given welldesigned base learners eg. Majority, Bayes, Average, Product. The Product and the Bayes rule assume independance of the base features. In most cases this is a pretty strong assumption and unrealistic. Compared to the Product rule, averaging the performance of the different features minimizes the errors of the base features. This however results in blurring and loss of information. The Majority scheme votes for the class most represented by the base features. If a majority of the base features are weak however, the response may be wrong most of the time.

(ii) Generative methods: These generate sets of base learners acting on the base learning algorithm or on the structure of the dataset, eg. Boosting. Adaptive boosting (AdaBoost) proposed by Freund and Schapire [10] has been used for improving the performance of a weak learning algorithm. AdaBoost generates classifiers sequentially for a certain number of trials and at each iteration the 
weights of the training dataset are changed based on the classifiers that were previously built. The final classifier is formed using a weighted voting scheme. Boosting however has been shown to suffer from over-fitting and noise sensitivity [11].

We propose a weighted feature combination scheme which like non-generative methods starts out with a set of well-designed base features or classifiers. The scheme however retains the flavor of AdaBoost in that the performance of the voters is evaluated against an objective truth. In a weighted feature combination scheme each feature's vote is factored by a weight depending on its importance in the final decision. The final decision is then determined by a weighted combination of the base learners. In order to learn each of the base feature's contribution, we need (i) an objective ground truth with which to quantitatively compare the performance of each weighted combination of classifiers, (ii) a means of estimating the confidence of each filter channel in making a decision and (iii) a cost or error function that we seek to minimize using different combinations of weights.

Our ensemble scheme belongs to the class of General Ensemble Methods (GEM). While the idea is not new [12, our method is novel in terms of the cost function employed. Based on the evaluation of this cost function, the weights of the different base learners can be fine tuned. Utilizing the ground truth associated with each input allows for optimization of the combination function which makes the scheme superior to the other non-generative methods described. For our problem, we seek to maximize the detection of the True Positive $(T P)$ area of the cancer while at the same time minimizing the False Positive $(F P)$ benign area. Hence, we define a cost $E^{(k)}$ which is a function $(\Phi)$ of both $T P$ and $F P$ and seek to find weights $\lambda_{\gamma}^{(k)}$ that minimize $E^{(k)}$ for a given training sample $k$.

$$
\sum_{\gamma=1}^{K} \lambda_{\gamma}^{(k)} f_{\gamma}^{(k)}=\mathcal{A}^{(k)} ; E^{(k)}=\Phi\left(\left\|\mathcal{A}_{a}{ }^{(k)}-\mathcal{A}_{m}{ }^{(k)}\right\|\right) \text { where } \Phi\left(\mathcal{A}_{a}, \mathcal{A}_{m}\right)=\frac{1}{1+\frac{F P\left(\mathcal{A}_{a}, \mathcal{A}_{m}\right)}{T P\left(\mathcal{A}_{a}, \mathcal{A}_{m}\right)}}
$$

where $\mathcal{A}_{m}{ }^{(k)}$ is the ground truth for image $k, \mathcal{A}^{(k)}$ is the combined Likelihood Scene generated from $K$ different classifiers $f_{\gamma}^{(k)}$, and $\mathcal{A}_{a}{ }^{(k)}$ is a binary mask obtained by thresholding $\mathcal{A}^{(k)}$.

One of the considerations in the choice of an optimization strategy is whether it is known a priori what the minima should be? In the case of our problem, it is not clear before hand what a good solution is, or what error is acceptable. Gradient Descent methods work on the premise that the solution will converge either to the minima or to some solution close to that. Brute Force methods on the other hand, are the only ones that are guaranteed to produce an optimal solution. However, the number of computations required increases exponentially with increase in the dimensionality of the problem. Assuming a step size of $\frac{1}{10}$ and 11 features, we would need $11^{10}$ computations to find the optimal weights for each of the $k$ training images. Even though the optimization is only done once during the training phase and off-line, this still represents a very large number of computations. 
We use a variant of the conventional Brute Force algorithm called the Hierarchical Brute Force algorithm which significantly reduces the number of iterations required. In this technique we initially start with a very coarse step size and obtain a set of weights which produces the minimum error. In the next iteration we search using a finer step size and only around the weights obtained in the previous step. The process is repeated until the weights do not change significantly. Using our Hierarchical Brute Force technique we find the smallest $E^{(k)}$ for some combination of $\lambda_{\gamma}^{(k)}$ for each sample $k$. The final weights $\lambda_{\gamma}$ are then obtained as the average of $\lambda_{\gamma}^{(k)}$ over all $k$ and used for all subsequent testing.

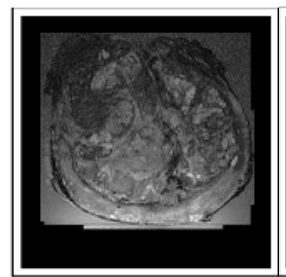

(a)

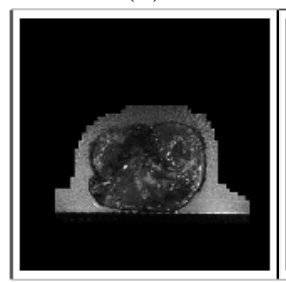

(e)

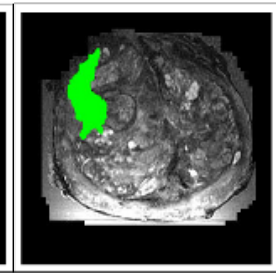

(b)

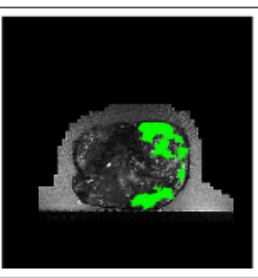

(f)

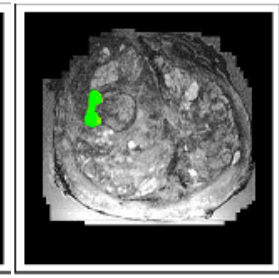

(c)

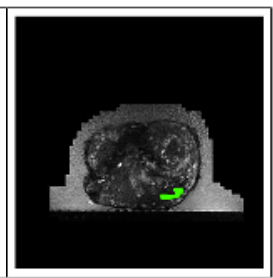

$(\mathrm{g})$

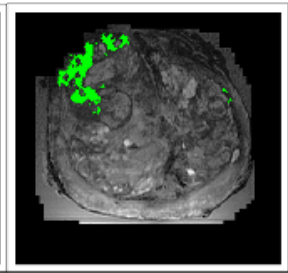

(d)

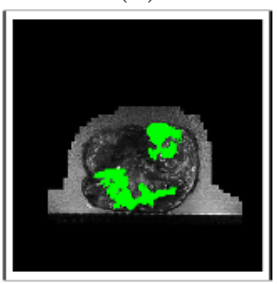

(h)

Fig. 1. (a),(e) Slices from 2 different MR scenes (b),(f) Ground truth derived from histology (c),(g) Expert's segmentation (d),(h) Result from GEM.

\section{Results}

A total of 33 slices from 5 different prostate glands were used for testing. We compared the result of our automated segmentation against an expert observer's manual segmentation of the tumor based on visual inspection of the MR data alone, as also the performance of the individual features. These results were quantitatively and qualitatively evaluated against the ground truth derived from the histology (refer to Section 31).

\subsection{Qualitative}

Figure 1 shows the result of our automated segmentation scheme on slices from two different glands. The manual segmentation of a large tumor by an expert observer (Figure 1(c)) based on visual inspection of the MR data (Figure 1(a)) is much smaller than the ground truth (Figure $1(b))$. The segmentation obtained by 
using our automated system (Figure1(d)) on the other hand is very similar in size and shape to the ground truth. For a slice from the second dataset (Figure 1(e)) the human expert missed most of the tumor in the upper right corner of the gland (Figure 1(g)) compared to the ground truth in Figure 1(f). Our segmentation method on the other hand picked out all the cancer with a small amount of false positive (Figure 1/(h)).

\subsection{Quantitative}

$\operatorname{Sensitivity~}\left(\frac{T P}{T P+F N}\right), \operatorname{PPV}\left(\frac{T P}{T P+F P}\right)$ and $\operatorname{Specificity~}\left(\frac{T N}{T N+F P}\right)$, (where $T N, F N$ refer to True Negative and False Negative areas respectively) were computed for the segmentations obtained for each of the 3D texture features, our GEM and an expert observer. The Likelihood Scenes corresponding to the individual features and the GEM were converted to binary scenes by comparing $P\left(\mathbf{x} \in \omega_{\varrho} \mid f_{\gamma}\right) \geq \delta$ for each voxel $\mathbf{x}$, where $\delta$ is a threshold between $0-1$. Figure 2(a) shows the ROC curves corresponding to the individual 3D texture features and our GEM. For the purposes of clarity, we have only shown one representative feature from each of the texture classes. As borne out by the area under the ROC curves in Figure $2(\mathrm{a})$, our GEM produced the best results. Figure $2(\mathrm{~b})$ shows a plot of the final $\alpha_{\gamma}$ for the $3 \mathrm{D}$ texture features. Note that the first-order statistical texture features have the largest weights, followed by the gradient features, the co-occurrence features and lastly the Gabor features.

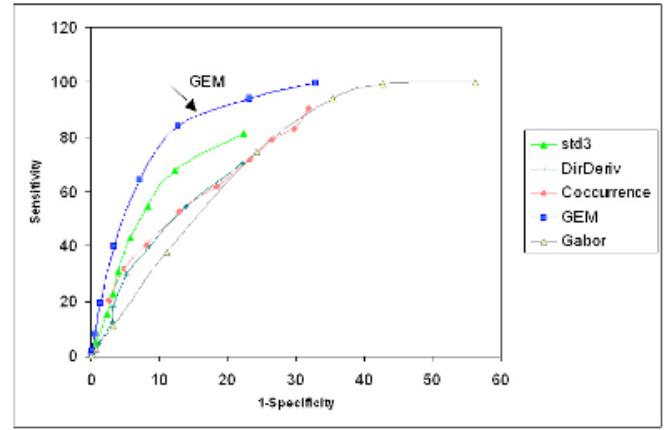

(a)

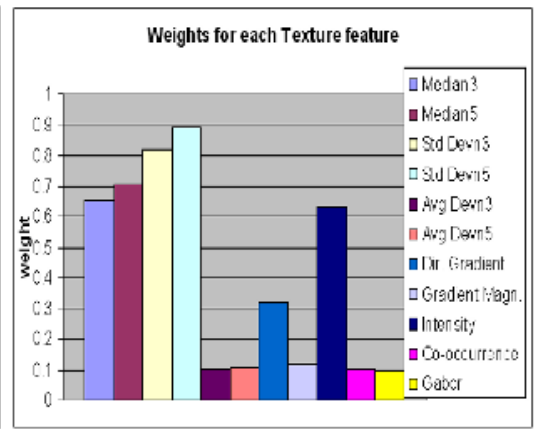

(b)

Fig. 2. (a) ROC Plot for 3D features \& GEM, (b) $\alpha_{\gamma}$ for each $3 \mathrm{D}$ features using 15 training samples.

In order to compare our automated segmentation with that of an expert observer, we divided our dataset into two groups. The large tumor group contained MR prostate slices in which the expert had been able to visually identify at least part of the cancer and the small tumor group in which the expert could not confidently identify any part of the cancer. Table 1 shows the Sensitivity (Sens.), PPV and Specificity (Spec.) for our GEM, the individual 3D texture features and a human observer. Our GEM outperformed the individual 3D features for 
Table 1. Comparing performance of individual features and human expert against $\operatorname{GEM}(\delta=0.5)$.

\begin{tabular}{|c|c|c|c|c|c|c|c|}
\hline Tumor & Texture & \multicolumn{2}{|c|}{ Statistical } & Grad- & Gabor & GEM & Expert \\
\cline { 3 - 8 } Size & Feature & $1^{\text {st }}$ & $2^{\text {nd }}$ & -ient & & & \\
\hline Large & Sens.\% & 25.34 & 69.93 & 29.64 & 39.86 & 41.35 & 36.41 \\
\cline { 2 - 8 } & PPV\% & 29.20 & 19.22 & 27.34 & 16.61 & 42.79 & 42.63 \\
\cline { 2 - 8 } & Spec.\% & 96.60 & 82.48 & 95.96 & 90.50 & 97.39 & 97.58 \\
\hline \multirow{3}{*}{ Small } & Sens.\% & 22.67 & 30.01 & 16.59 & 13.05 & 40.11 & 0 \\
\cline { 2 - 8 } & PPV & 6.21 & 3.38 & 4.86 & 3.02 & 10.02 & 0 \\
\cline { 2 - 7 } & Spec.\% & 95.651 .11 & 95.67 & 88.41 & 95.73 & 0 \\
\hline
\end{tabular}

Table 2. Effect of training on $\alpha_{\gamma}(\delta=0.4)$.

\begin{tabular}{|c|c|c|c|}
\hline $\begin{array}{c}\text { Training } \\
\text { Samples }\end{array}$ & $\begin{array}{c}\text { PPV } \\
\%\end{array}$ & $\begin{array}{c}\text { Sens. } \\
\%\end{array}$ & $\begin{array}{c}\text { Spec. } \\
\%\end{array}$ \\
\hline 10 & 21.201 & 58.684 & 93.892 \\
\hline 15 & 21.142 & 58.681 & 93.908 \\
\hline 25 & 20.474 & 58.685 & 93.941 \\
\hline 30 & 21.201 & 58.684 & 93.892 \\
\hline Std. Devn & 0.354 & 0.001 & 0.022 \\
\hline
\end{tabular}

both the large and small tumors in terms of Sensitivity, PPV and Specificity. Further, our GEM significantly outperformed the expert in terms of Sensitivity and PPV, while the differences in Specificity were found to be not statistically significant. While the results for the small tumors may not appear as remarkable as those for the visible ones, it should be borne in mind that the average size of these smaller tumors expressed as a percentage of the slice area was only $0.24 \%$ compared to $7.29 \%$ for the larger tumors (30 times larger than the tumors that the expert could not visually identify). In light of this, a Sensitivity of $40 \%$ and PPV of over $10 \%$ is remarkable.

To analyze the sensitivity of the $\alpha_{\gamma}$ on the training data, we randomly selected different sets of training samples from our database and used them for computing the feature weights. Table 2 shows the Sensitivity (Sens.), Specificity (Spec.) and PPV for our GEM for 10, 15, 25 and 30 training samples. The small standard deviations for these error metrics for different sets of training samples shows the robustness of our GEM to training.

\section{Conclusions}

In this paper we have presented a fully automated segmentation system for detecting prostatic adenocarcinoma from high resolution MR using an optimal feature ensemble of 3D statistical, gradient and Gabor features. Our GEM outperformed the individual texture features and a human observer in terms of Sensitivity and PPV for large tumors. It was also able to detect tumors that could not be visually detected by an expert observer. Further the ensemble was 
found to be robust to the number of training samples used. Among the 3D features, first order statistical features performed the best. It has been shown that inhomogeneity correction tends to increase noise variance in MR images 13 . The superior performance of the first order statistics could be explained by the fact that they are more robust to noise than higher order features. The poor performance of the Gabor filter could reflect the differences in the pathologic homogeneity of tumors. In large tumors, the nodules are homogeneous while small tumors are composed of varying mixtures of benign and malignant glands. While our GEM is optimal in terms of reducing the cost function, unlike Adaboost [10] it still does not guarantee maximum feature separability. We intend to pursue this area in future work.

\section{References}

1. V. Kovalev, M. Petrou, Y. Bondar, Texture Anisotropy in 3-D Images, IEEE Trans. on Image Proc., 1999, vol. 8[3], pp. 34-43.

2. M. Schiebler, M. Schnall, et al., Current Role of MR Imaging in staging of adenocarcinoma of the prostate, Radiology, 1993, vol. 189[2], pp. 339-352.

3. Gnadt, W., Manolakis D., et al., "Classification of prostate tissue using neural networks", Int. Joint Conf. on Neural Net., 1999, vol. 5, pp. 3569-72

4. R. Haralick, K. Shanmugan, I. Dinstein, "Textural Features for Image Classification", IEEE Trans. Syst. Man. Cybern., 1973, vol. SMC-3, pp. 610-621.

5. A. Madabhushi, J. Udupa, "Interplay of Intensity Standardization and Inhomogeneity Correction in MR Image Analysis", SPIE, 2003, vol. 5032, pp. 768-779.

6. A. Houston, S. Premkumar, D. Pitts, "Prostate Ultrasound Image Analysis", IEEE Symp. on Computer-Based Med. Syst., pp. 94-101, 1995.

7. D. Basset, Z. Sun, et al., "Texture Analysis of Ultrasonic Images of the Prostate by Means of Co-Occurrence Matrices", Ultrasonic Imaging, 1993, vol. 15, pp. 218-237.

8. A. Jain, F. Farrokhnia, "Unsupervised Texture Segmentation Using Gabor Filters", Pattern Recog., 1991, vol. 24[12], pp. 1167-1186.

9. R. Duda, P. Hart, Pattern Classification and Scene Analysis, New York Wiley, 1973.

10. Y. Freund, R. Schapire, "Experiments with a new Boosting Algorithm", National Conference on Machine Learning, 1996, pp. 148-156.

11. T. Dietterich, "Ensemble Methods in Machine Learning", Workshop on Multiple Classifier Systems, 2000, pp. 1-15.

12. M. Perrone, "Improving regression estimation", Ph.D. Thesis, Dept. of Physics, Brown University, 1993.

13. A. Montillo, J. Udupa, L. Axel, D. Metaxas, "Interaction between noise suppression \& inhomogeneity correction", SPIE, 2003, vol. 5032, pp. 1025-1036. 